Published in final edited form as:

Int J Hematol. 2011 February ; 93(2): 206-212. doi:10.1007/s12185-010-0747-x.

\title{
Targeted IV busulfan and fludarabine followed by post-allogeneic hematopoietic cell transplantation rituximab demonstrate encouraging activity in CD20+ lymphoid malignancies without increased risk of infectious complications
}

\author{
Joseph Pidala, \\ Department of Blood and Marrow Transplantation, Moffitt Cancer Center, 12902 Magnolia Drive, \\ FOB-3, Tampa, FL 33612, USA. Department of Oncological Sciences, Moffitt Cancer Center, \\ University of South Florida, Tampa, FL, USA
}

Jaime Roman-Diaz,

Department of Blood and Marrow Transplantation, Moffitt Cancer Center, 12902 Magnolia Drive, FOB-3, Tampa, FL 33612, USA

\section{Jongphil Kim,}

Department of Oncological Sciences, Moffitt Cancer Center, University of South Florida, Tampa, FL, USA. Department of Biostatistics, Moffitt Cancer Center, Tampa, FL, USA

\section{Taiga Nishihori,}

Department of Blood and Marrow Transplantation, Moffitt Cancer Center, 12902 Magnolia Drive, FOB-3, Tampa, FL 33612, USA

\section{Janelle Perkins,}

Department of Blood and Marrow Transplantation, Moffitt Cancer Center, 12902 Magnolia Drive, FOB-3, Tampa, FL 33612, USA

\section{Cheryl Tate,}

Department of Blood and Marrow Transplantation, Moffitt Cancer Center, 12902 Magnolia Drive, FOB-3, Tampa, FL 33612, USA

\section{Jose L. Ochoa-Bayona,}

Department of Blood and Marrow Transplantation, Moffitt Cancer Center, 12902 Magnolia Drive, FOB-3, Tampa, FL 33612, USA. Department of Oncological Sciences, Moffitt Cancer Center, University of South Florida, Tampa, FL, USA

Teresa Field,

Correspondence to: Mohamed A. Kharfan-Dabaja.

Present Address: M. A. Kharfan-Dabaja, Division of Hematology-Oncology-BMT, Department of Internal Medicine, American University of Beirut, P.O. Box 11-0236, Riad El Solh, Beirut 1107 2020, Lebanon

J. Pidala and J. Roman-Diaz contributed equally to this work.

Conflict of interest

The authors report the following funding sources which have relevance to the work described here: Claudio Anasetti, MD, and Janelle Perkins, PharmD, have research funding from Protein Design Labs BioPharma for the conduct of research involving busulfan and fludarabine as conditioning therapy prior to allogeneic transplantation. 
Department of Blood and Marrow Transplantation, Moffitt Cancer Center, 12902 Magnolia Drive, FOB-3, Tampa, FL 33612, USA. Department of Oncological Sciences, Moffitt Cancer Center, University of South Florida, Tampa, FL, USA. Department of Biostatistics, Moffitt Cancer Center, Tampa, FL, USA

\section{Hugo F. Fernandez,}

Department of Blood and Marrow Transplantation, Moffitt Cancer Center, 12902 Magnolia Drive, FOB-3, Tampa, FL 33612, USA. Department of Oncological Sciences, Moffitt Cancer Center, University of South Florida, Tampa, FL, USA

\section{Marcie Tomblyn,}

Department of Blood and Marrow Transplantation, Moffitt Cancer Center, 12902 Magnolia Drive, FOB-3, Tampa, FL 33612, USA. Department of Oncological Sciences, Moffitt Cancer Center, University of South Florida, Tampa, FL, USA

\section{Ernesto Ayala,}

Department of Blood and Marrow Transplantation, Moffitt Cancer Center, 12902 Magnolia Drive, FOB-3, Tampa, FL 33612, USA. Department of Oncological Sciences, Moffitt Cancer Center, University of South Florida, Tampa, FL, USA

Claudio Anasetti, and

Department of Blood and Marrow Transplantation, Moffitt Cancer Center, 12902 Magnolia Drive, FOB-3, Tampa, FL 33612, USA. Department of Oncological Sciences, Moffitt Cancer Center, University of South Florida, Tampa, FL, USA

\section{Mohamed A. Kharfan-Dabaja}

Department of Blood and Marrow Transplantation, Moffitt Cancer Center, 12902 Magnolia Drive, FOB-3, Tampa, FL 33612, USA. Department of Oncological Sciences, Moffitt Cancer Center, University of South Florida, Tampa, FL, USA

\section{Abstract}

We examined pharmacokinetic-targeted IV busulfan $\left(75-170 \mathrm{mg} / \mathrm{m}^{2}\right.$, with target AUC of $3500-$ $6000 \mu \mathrm{mol} \mathrm{min})$ and fludarabine $\left(40 \mathrm{mg} / \mathrm{m}^{2}\right) \times 4$ days with rituximab (t-IV Bu/Flu + rituximab) $375 \mathrm{mg} / \mathrm{m}^{2}$ on days +1 and +8 followed by allogeneic hematopoietic cell transplantation in 19 patients (median age 56, range 35-68 years) with CD20+ lymphoid malignancies. Median time to neutrophil and platelet engraftment was 15 and 12 days. The cumulative incidence of grade II-IV acute graft-versus-host disease (GVHD) was 58\% (95\% confidence interval, CI 39-85\%), and chronic GVHD was 50\% (95\% CI 28-88\%). With a median follow up of 7 (range 1-31) months, overall response was observed in 15, and stable or progressive disease in 4 . Overall survival at 1 year was $67 \%$. Engraftment, chimerism, and infectious complications did not differ significantly from a contemporaneous non-rituximab containing comparator group. The addition of rituximab $375 \mathrm{mg} / \mathrm{m}^{2}$ to t-IV Bu/Flu does not appear to adversely affect engraftment, donor chimerism, or increase the risk of infectious complications.

\section{Keywords}

Allogeneic hematopoietic cell transplantation; Rituximab; Busulfan; Fludarabine 


\section{Introduction}

Therapeutic advances have allowed improved outcomes in patients receiving allogeneic hematopoietic cell transplantation (AHCT) for the treatment of lymphoid malignancies [14]. However, primary disease relapse remains a significant cause of failure after AHCT, particularly for those with more advanced disease stage or limited response to salvage chemotherapy prior to AHCT. Rituximab, a chimeric murine/human anti-CD20 monoclonal antibody, has revolutionized medical therapy of CD20 positive lymphoid malignancies [5, $6]$.

Emerging data suggest that the addition of rituximab to programs of conditioning therapy and AHCT may also enhance disease control after hematopoietic cell transplantation (HCT). Khouri et al. originally reported outcomes following a regimen of fludarabine, cyclophosphamide, and rituximab $\left(375 \mathrm{mg} / \mathrm{m}^{2}\right.$ on day -6 and $1000 \mathrm{mg} / \mathrm{m}^{2}$ on days $+1,+8$, and +15 post-allografting $)$ in 20 patients with follicular NHL $(N=18)$ or small lymphocytic leukemia $(N=2)$. Actuarial probability of being alive and in remission at 2 years was $84 \%$ (95\% confidence interval, CI 57-94\%) [7]. In a later series, 47 patients with relapsed follicular lymphoma were treated with fludarabine, cyclophosphamide and rituximab [375 $\mathrm{mg} / \mathrm{m}^{2}$ (day -13 ) and $1000 \mathrm{mg} / \mathrm{m}^{2}$ (days $-6,+1$, and +8 )]. Estimated progression-free survival (PFS) and overall survival (OS) at a median of 60 (19-94) months were $83 \%$ (95\% CI 69-91\%) and 85\% (95\% CI 85-95\%) [8], respectively. In both series, the incidence of grade II-IV acute graft-versus-host disease (aGVHD) (20 and 11\%, respectively) was low, but the cumulative incidence of chronic GVHD (cGVHD) ranged from 60 to $64 \%$. Glass et al. [9] reported a 1-year OS of $49 \%$ in a series of 59 patients with aggressive lymphoma treated with fludarabine, busulfan, cyclophosphamide, and rituximab (4 doses starting at days +21 and +175 post-allografting). Evidence also suggests that prior rituximab therapy may result in significantly lower incidence of aGVHD [10] as well as supports its activity in the treatment of glucocorticoid-refractory cGVHD [11-13]. Therefore, evidence to date suggests that the incorporation of rituximab in conditioning regimen platforms for lymphoid malignancies may contribute to both primary disease control as well as mitigate GVHD risk following AHCT. We hereby evaluate the safety and feasibility of an approach of pharmacokinetic-targeted IV busulfan $\left(130-145 \mathrm{mg} / \mathrm{m}^{2}\right)$ plus fludarabine $\left(40 \mathrm{mg} / \mathrm{m}^{2}\right) \times 4$ days (t-IV Bu/Flu) and post-AHCT rituximab at $375 \mathrm{mg} / \mathrm{m}^{2}$ on days +1 and +8 .

\section{Methods}

A retrospective review of patient records was performed under the approval of the University of South Florida's Institutional Review Board. From allogeneic hematopoietic cell transplant procedures performed at the Moffitt Cancer Center, all patients with CD20+ lymphoid malignancies who were treated with a myeloablative conditioning regimen of pharmacokinetic-targeted IV busulfan (75-170 mg/m², with target AUC of 3500-6000 $\mu \mathrm{mol}$ $\mathrm{min}$ ) and fludarabine $\left(40 \mathrm{mg} / \mathrm{m}^{2}\right) \times 4$ days (t-IV Bu/Flu) and rituximab at $375 \mathrm{mg} / \mathrm{m}^{2}$ on days +1 and +8 post-HCT were identified. A contemporaneous comparator group of all $\mathrm{CD} 20+$ lymphoid malignancies conditioned with t-IV Bu/Flu without post-transplantation rituximab was also identified. The conditioning regimen consisted of fludarabine, $40 \mathrm{mg} / \mathrm{m}^{2}$ infused over $30 \mathrm{~min}$ on days -6 to -3 , followed by intravenous busulfan, $75-170 \mathrm{mg} / \mathrm{m}^{2}$ 
over $4 \mathrm{~h}$ daily on the same days $[14,15]$. Busulfan $(\mathrm{Bu})$ PK-samples were obtained on day -6 and analyzed by mass spectrometry; on days -4 and -3 , the BU dose was adjusted to target an average AUC of 3500-6000 $( \pm 10 \%) \mu \mathrm{mol}$ min for each of the 4 days. Baseline characteristics gathered in all cases included the following: age at the time of AHCT; gender; diagnosis; remission status at the time of HCT; aGVHD prophylaxis regimen utilized; stem cell source was peripheral blood mobilized stem cells in all cases; donor source and degree of HLA matching; conditioning regimen, rituximab administered, and the use of anti-thymocyte globulin (ATG) in those cases using a HLA-mismatched donor; CMV serostatus of donor and recipient; and date of transplant. Outcomes of interest included the following: time to neutrophil (defined by the first of three successive days with an absolute neutrophil count (ANC) of greater than 500/ $\mathrm{LL}$ ) and platelet (defined by the first of three successive days with a non-transfused platelet count of greater than 20000/ $\mu \mathrm{L}$ ) engraftment; donor CD3, CD33 chimerism in the peripheral blood, and unsorted donor bone marrow chimerism at days 30, 90, 180, and 360 by PCR; maximal grade of aGVHD reached in follow up, according to modified Glucksberg criteria [16]; maximal grade of cGVHD reached in follow up in those at risk surviving beyond day 100, according to proposed NIH consensus definitions [17]; disease response at days 30, 90, 180, and 360 post-HCT; dates of death or last follow up; CMV reactivation according to recipient/donor serostatus matching; the occurrence of infectious complications, classified as bacterial, invasive fungal, or viral infections other than CMV reactivation; and absolute lymphocyte repopulation after HCT at days 30, 60, 90, 180, 360, 2 years, and 3 years. B lymphocyte data were abstracted at these time points if available.

\subsection{Statistical analysis}

Baseline characteristics were summarized using descriptive statistics including median for continuous measures, and frequencies for categorical variables. Comparisons between rituximab and non-rituximab groups were made using Fisher's exact test for categorical baseline variables, and Wilcoxon's Rank-Sum test for continuous variables. Time to neutrophil and platelet engraftment was calculated using Kaplan-Meier method and compared between groups by log-rank test. After neutrophil and platelet engraftment was achieved, the following was collected in all cases: the occurrence of ANC $<1000 / \mu \mathrm{L}$ within 100 days post-AHCT; total number of days with ANC $<1000 / \mu \mathrm{L}$ within 100 days postAHCT; number of doses of G-CSF administered within 100 days post-AHCT; the occurrence of ANC < 1000/ $\mu \mathrm{L}$ after day 100 post-AHCT; number of doses of G-CSF administered beyond day 100; number of days with platelet count $<20000 / \mu \mathrm{L}$; and number of platelet transfusions received. Comparisons of these outcomes across groups utilized Fisher's exact test for categorical outcomes, and Wilcoxon's Rank-Sum test for continuous outcomes. Median (range) values for donor chimerism were reported. Donor chimerism was compared across groups by Wilcoxon Rank-Sum test. The cumulative incidence of aGVHD and cGVHD was estimated accounting for competing risk events. Best disease response by time of last follow up was reported, categorized as proportion with progressive disease, stable disease, partial remission, or complete remission. OS was estimated from the date of HCT utilizing the Kaplan-Meier method. Median (range) values for absolute lymphocyte counts were reported for each group; comparison across groups utilized Wilcoxon Rank- 
Sum test. The proportion with CMV reactivation, bacterial, fungal, and viral infections was described for each group, and comparison is made using Fisher's exact test.

\section{Results}

A total of 19 patients were identified by retrospective review to have been conditioned with $\mathrm{t}-\mathrm{IV} \mathrm{Bu} /$ Flu followed by rituximab $375 \mathrm{mg} / \mathrm{m}^{2}$ IV on days +1 and +8 post-HCT for the treatment of CD20 positive lymphoid malignancies from AHCT procedures performed from 2005 to 2010 at the center. A contemporaneous group of 19 patients was identified to have been conditioned with t-IV Bu/Flu without post-transplant rituximab for the treatment of CD20 positive lymphoid malignancies from 2004 to 2009 at the center. The use of rituximab was guided at the discretion of the treating transplant physician, and no systematic selection criteria were applied. Baseline characteristics are summarized in Table 1. These groups consisted of a heterogeneous array of representative CD20 positive lymphoid malignancies. While all had demonstrated chemotherapy responsiveness to salvage therapy prior to allogeneic HCT in the t-IV Bu/Flu + rituximab group, one in the t-IV Bu/Flu group was transplanted with refractory disease.

The conditioning regimen in all cases consisted of fludarabine, $40 \mathrm{mg} / \mathrm{m}^{2}$ infused over 30 min on days -6 to -3 , followed by intravenous busulfan, $75-170 \mathrm{mg} / \mathrm{m}^{2}$ over $4 \mathrm{~h}$ daily on the same days. Busulfan (Bu) PK-samples were obtained on day -6 and analyzed by mass spectrometry; on days -4 and -3 , the BU dose was adjusted to target an average AUC of $3500-6000( \pm 10 \%) \mu \mathrm{mol}$ min for each of the 4 days; the reduced busulfan target AUC was selected according to transplant physician discretion. Target AUC in the rituximab group was $3500(n=9), 5300(n=9)$, and $6000(n=1)$. Median actual AUC after the first dose was 3874 (range 2998-5028) $\mu \mathrm{M}$ min for the $3500 \mu \mathrm{M}$ min target subgroup, 5510 (range 4315-7239) $\mu \mathrm{M}$ min for the $5300 \mu \mathrm{M}$ min target subgroup, and $5964 \mu \mathrm{M}$ min for one patient with the 6000 AUC target. Daily busulfan doses were adjusted to achieve the target AUC averaged over the 4 days. The median total busulfan dose required to achieve the target AUC was 378 (range 308-520) $\mathrm{mg} / \mathrm{m}^{2}$ for the AUC 3500 target subgroup, 520 (range 428-632) $\mathrm{mg} / \mathrm{m}^{2}$ for the AUC 5300 target subgroup, and $680 \mathrm{mg} / \mathrm{m}^{2}$ in one case with the 6000 AUC target. In the non-rituximab group, the target AUC was $3500(n=5), 5300(n=12)$, and $6000(n=2)$. The median actual AUC after first dose was 4253 (range 2912-5090) $\mu \mathrm{M}$ min in the target AUC 3500 group, 5400 (range 3797-6478) $\mu \mathrm{M}$ min in the target AUC 5300 group, and ranged from 5105 to $8015 \mu \mathrm{M}$ min for the two patients in the target AUC 6000 group. Daily busulfan doses were adjusted to achieve the intended target AUC averaged over the 4 days. The median total busulfan dose required to achieve the target AUC was 384 (range 312-458) mg/m² in the AUC 3500 subgroup, 526 (range 490-704) $\mathrm{mg} / \mathrm{m}^{2}$ in the AUC 5300 subgroup, and ranged from 508 to $688 \mathrm{mg} / \mathrm{m}^{2}$ in the AUC 6000 target subgroup.

The administration of rituximab did not appear to result in any immediate unexpected toxicity or interference with administration of standard post-AHCT therapy. The median time to sustained neutrophil engraftment was 15 (range 6-20) days, and median time to sustained platelet engraftment was 12 (range 9-18) days. Hematopoietic engraftment was not delayed compared to those conditioned with t-IV Bu/Flu without post-transplant rituximab (Fig. 1). One patient with CLL who received a matched-related donor allograft 
required infusion of donor lymphocytes due to a CD3 donor engraftment of $10 \%$ at day +30 measured by STR/PCR on peripheral blood with improvement to $100 \%$ afterward. A second patient with CLL who received a mismatched $(\mathrm{C} \mathrm{Ag})$ unrelated donor allograft developed late selective graft failure of the donor CD3 compartment on day +167 post-AHCT followed by disease relapse requiring DLI infusion.

After attainment of neutrophil and platelet engraftment, there was no significant difference between the t-IV Bu/Flu + rituximab and t-IV Bu/Flu groups, respectively, for the following: proportion with ANC < 1000/ $\mu \mathrm{L}$ within 100 days post-AHCT (6/19 vs. 11/19, $p=0.195$ ); total number of days with ANC $<1000 / \mu \mathrm{L}$ within 100 days (mean 2.9 vs. $3.4, p=0.25$ ); number of G-CSF doses administered within 100 days (mean 1.3 vs. 1.4, $p=0.96$ ); occurrence of ANC < 1000/ $\mu \mathrm{L}$ beyond day 100 (9/19 vs. 6/19, $p=0.25)$; number of doses of G-CSF administered post day 100 (mean 4.8 vs. $2.4, p=0.28$ ); number of days with platelet count $<20000 / \mu \mathrm{L}$ (mean 1.5 vs. 2.2, $p=0.8$ ); and number of platelet transfusions (mean 1.2 vs. 1.9, $p=0.82$ ). The median (range) values for donor chimerism assessed by STR/PCR were the following: At day 30 post-AHCT, peripheral blood CD3 was 100\% (10-100\%), CD33 was $100 \%$ (79-100\%), and unsorted bone marrow donor chimerism was 98\% (84$100 \%$ ). At day 90 post-HCT, peripheral blood CD3 was 94\% (73-100\%), CD33 was exclusively $100 \%$, and unsorted bone marrow donor chimerism was $95 \%$ (88-100\%). Donor chimerism at each point did not significantly differ compared to the non-rituximab group (Table 2). The cumulative incidence of grade II-IV aGVHD was 58\% (95\% CI 39-85\%), and the cumulative incidence of any grade cGVHD was 50\% (95\% CI 28-88\%). The corresponding data for the non-rituximab cohort were 66\% (95\% CI 47-93\%) and 58\% (95\% CI 39-89\%), respectively.

Clinical disease response was graded at standard time points, including days 30, 90, 180, 360 , and then annually if applicable. The regimen demonstrated encouraging activity in a group of largely advanced, but chemotherapy responsive lymphoid malignancies. The rituximab cohort had a median follow up of 7 (range 1-31) months. By day 100 post-HCT, overall response occurred in 15/19, and complete response in 11/19. Of those transplanted with active disease (not in complete remission at the time of HCT), by day 100 overall response was observed in 9/14, and complete response in 6/14. By time of last follow up, overall response was observed in 15 , and stable or progressive disease in 4 . In the nonrituximab cohort, by day 100 overall response was observed in 15/19, and complete response in 12/19. Median OS from date of transplant was not reached (Fig. 2). One-year OS was $67 \%$. OS did not significantly differ according to remission status at the time of AHCT (logrank comparison for complete vs. partial remission, $p=0.892$ ). Causes of death were the following: refractory $\operatorname{GVHD}(n=1)$, sepsis/multi-organ failure $(n=2)$, and relapsed disease $(n=1)$.

Absolute lymphocyte counts were measured at serial designated time points. As demonstrated in Table 3, there was absolute lymphopenia, which persisted through 1 year. The median absolute lymphocyte count was significantly lower at day 90 in the t-IV Bu/Flu + rituximab group; lymphocyte recovery did not significantly differ compared to the nonrituxan group at any other time points. In the limited number of patients with data at 2 years post-HCT ( $n=4)$, absolute lymphocyte counts uniformly had returned to the normal range 
(median 1.4, range 1.2-2k/ $/ \mu \mathrm{L}$ ). In those with available data on B lymphocyte repopulation ( $n$ $=5$ ), $\mathrm{B}$ lymphopenia persisted throughout first year.

All instances of infectious complications from date of transplant until death or last follow up were collected for each patient. In total, CMV reactivation was detected in 6 cases by weekly surveillance of peripheral blood CMV PCR, and was preemptively treated to resolution in all cases. Reactivation according to recipient/donor serostatus for the t-IV $\mathrm{Bu} / \mathrm{Flu}+$ rituximab group was the following: negative/negative 0/6; negative/positive 1/4; positive/negative $3 / 5$; and positive/positive $2 / 4$. CMV reactivation according to recipient/ donor serostatus in the t-IV Bu/Flu group was the following: negative/negative 0/7; negative/ positive 0/1; positive/negative 3/5; positive/positive 4/6. Other bacterial, viral, and invasive fungal infections are reported in Table 4.

\section{Discussion}

The addition of rituximab has improved efficacy of chemotherapy programs in the treatment of CD20+ lymphoid malignancies. Emerging data suggest a beneficial role for the addition of rituximab to treatment programs in AHCT. However, the use of rituximab post-AHCT poses potential risk of lymphopenia and resultant immune suppression. In this retrospective series, we aimed at exploring the safety and efficacy of the addition of rituximab $375 \mathrm{mg} / \mathrm{m}^{2}$ on days +1 and +8 after a regimen of pharmacokinetic-targeted IV busulfan $\left(75-170 \mathrm{mg} / \mathrm{m}^{2}\right.$, with target AUC of 3500-6000 $\mu \mathrm{mol} \mathrm{min}$ ) and fludarabine $\left(40 \mathrm{mg} / \mathrm{m}^{2}\right) \times 4$ days (t-IV Bu/ Flu) and HCT for CD20+ lymphoid malignancies [18].

Overall, the addition of rituximab did not impose any unexpected toxicity post-AHCT. Neutrophil and platelet engraftment kinetics and establishment of donor chimerism did not appear to be adversely affected by the addition of rituximab to this treatment program. Outside of lower values at day 90 post-AHCT, the rituximab group did not have significantly lower absolute lymphocyte counts compared to the non-rituximab comparator group. Here, we also demonstrate that the overall incidence of bacterial, viral, and invasive fungal infections did not significantly differ in the t-IV Bu/Flu + rituximab versus the t-IV Bu/Flu group. We did not observe any other late complications, and there have been no cases of progressive multi-focal leukoencephalopathy. We recognize the limitations inherent in this series, namely its retrospective nature, the small number of total patients, the diversity of histologic entities, and the relatively short follow up. Importantly, the addition of rituximab to the conditioning regimen was not based on systematic selection criteria, but rather the discretion of the transplant physician. Based on this limitation, we specifically have not drawn conclusions on the comparative efficacy of these regimens. The emphasis of this comparison is rather to examine the effect of rituximab on engraftment, donor chimerism, and infectious complications. The comparison of infectious complications and other clinical outcomes is bolstered by the contemporaneous nature of these two groups, as similar supportive care and infectious prophylaxis were provided during this period.

The data show an overall incidence of cGVHD which is comparable to that reported in prior series employing rituximab in allogeneic HCT programs, ranging from 60 to 64\% (extensive cGVHD in 36\%) after fludarabine, cyclophosphamide, and rituximab as per Khouri et al. [7, 
8]. However, 58\% of patients in our series experienced grade II-IV aGVHD, which exceeds rates of aGVHD seen in this literature likely due to the fact that the majority of patients in our series received allografts from HLA-mismatched or unrelated donors $[7,8,10]$. We have not drawn further conclusions regarding the incidence of aGVHD or cGVHD across the rituximab and non-rituximab groups in our series given the retrospective nature of the study, and heterogeneous transplant conditions and GVHD prophylaxis. These and other limitations circumscribe direct comparisons between these data and published series, including differences in established risk factors for the development of aGVHD and cGVHD. Further work, including the conduct of prospective trials powered to address the magnitude of benefit provided in GVHD prevention with the addition of rituximab to conditioning therapy and allogeneic HCT, is needed to draw more firm conclusions in this regard.

Finally, acknowledging the limitations of this sample, these early data suggest the clinical activity of this regimen in the treatment of CD20+ lymphoid malignancies. With the limited follow up to date, response rates are encouraging, and estimated OS at 1 year is $67 \%$. These results are especially notable, given the largely advanced disease at the time of transplant. However, it should be noted that all of the patients in the t-IV Bu/Flu + rituximab group had chemotherapy responsive disease at the time of HCT. It is clear from allied literature that those with chemotherapy refractory disease suffer worse outcomes, and these data do not address whether the addition of rituximab improves upon historical disease control achieved with non-rituximab containing allogeneic HCT approaches in those with chemotherapy refractory disease. Additionally, conclusions regarding the efficacy of this approach for particular subtypes of CD20 positive lymphoid malignancies are limited by the heterogeneous and small sample represented here. Further conclusions on the effectiveness of this approach of t-IV Bu/Flu with rituximab $375 \mathrm{mg} / \mathrm{m}^{2}$ on days +1 and +8 post-AHCT will require systematic study of a larger sample, as well as more mature follow-up time to discern the durability of these responses.

\section{References}

1. Kharfan-Dabaja MA, Anasetti C, Santos ES. Hematopoietic cell transplantation for chronic lymphocytic leukemia: an evolving concept. Biol Blood Marrow Transpl. 2007; 13:373-85.

2. Ghielmini M, Zucca E. How I treat mantle cell lymphoma. Blood. 2009; 114:1469-76. [PubMed: 19556426]

3. Khouri IF. Reduced-intensity regimens in allogeneic stem-cell transplantation for non-Hodgkin lymphoma and chronic lymphocytic leukemia. Hematol Am Soc Hematology Educ Program. 2006:390-7.

4. van Besien K. Allogeneic stem cell transplantation in follicular lymphoma: recent progress and controversy. Hematology Am Soc Hematol Educ Program. 2009:610-8. [PubMed: 20008246]

5. Bello C, Sotomayor EM. Monoclonal antibodies for B-cell lymphomas: rituximab and beyond. Hematology Am Soc Hematol Educ Program. 2007:233-42. [PubMed: 18024635]

6. Cheson BD, Leonard JP. Monoclonal antibody therapy for B-cell non-Hodgkin's lymphoma. N Engl J Med. 2008; 359:613-26. [PubMed: 18687642]

7. Khouri IF, Saliba RM, Giralt SA, et al. Nonablative allogeneic hematopoietic transplantation as adoptive immunotherapy for indolent lymphoma: low incidence of toxicity, acute graft-versus-host disease, and treatment-related mortality. Blood. 2001; 98:3595-9. [PubMed: 11739162] 
8. Khouri IF, McLaughlin P, Saliba RM, et al. Eight-year experience with allogeneic stem cell transplantation for relapsed follicular lymphoma after nonmyeloablative conditioning with fludarabine, cyclophosphamide, and rituximab. Blood. 2008; 111:5530-6. [PubMed: 18411419]

9. Glass B. Rituximab for graft-versus-host disease prophylaxis after allogeneic stem cell transplantation given as treatment of high risk relapse of aggressive lymphoma: results of a randomized phase II study. Blood. 2008; 112:689-90.

10. Ratanatharathorn V, Logan B, Wang D, et al. Prior rituximab correlates with less acute graftversus-host disease and better survival in B-cell lymphoma patients who received allogeneic peripheral blood stem cell transplantation. Br J Haematol. 2009; 145:816-24. [PubMed: 19344418]

11. Ratanatharathorn V, Ayash L, Reynolds C, et al. Treatment of chronic graft-versus-host disease with anti-CD20 chimeric monoclonal antibody. Biol Blood Marrow Transpl. 2003; 9:505-11.

12. Cutler C, Miklos D, Kim HT, et al. Rituximab for steroid-refractory chronic graft-versus-host disease. Blood. 2006; 108:756-62. [PubMed: 16551963]

13. Kharfan-Dabaja MA, Mhaskar AR, Djulbegovic B, Cutler C, Mohty M, Kumar A. Efficacy of rituximab in the setting of steroid-refractory chronic graft-versus-host disease: a systematic review and meta-analysis. Biol Blood Marrow Transpl. 2009; 15:1005-13.

14. de Lima M, Couriel D, Thall PF, et al. Once-daily intravenous busulfan and fludarabine: clinical and pharmacokinetic results of a myeloablative, reduced-toxicity conditioning regimen for allogeneic stem cell transplantation in AML and MDS. Blood. 2004; 104:857-64. [PubMed: 15073038]

15. Pidala J, Kim J, Anasetti C, et al. Targeted i.v. BU and fludarabine (t-i.v. BU/Flu) provides effective control of AML in adults with reduced toxicity. Bone Marrow Transpl. 2010 [Epub ahead of print].

16. Przepiorka D, Weisdorf D, Martin P, et al. 1994 consensus conference on acute GVHD grading. Bone Marrow Transpl. 1995; 15:825-8.

17. Filipovich AH, Weisdorf D, Pavletic S, et al. National Institutes of Health consensus development project on criteria for clinical trials in chronic graft-versus-host disease. I. Diagnosis and staging working group report. Biol Blood Marrow Transpl. 2005; 11:945-56.

18. Kharfan-Dabaja M, Tate C, Perkins J, Fernandez H, Field T, Ayala E, Perez L, Raychaudhuri J, Alsina M, Ochoa-Bayona L, Sullivan D, Brand L, Poling W, Anasetti C. Rituximab is feasible to administer to allograft recipients with advanced CD20+ malignancies and does not affect timely hematopoietic engraftment. Biol Blood Marrow Transpl. 2008; 14:121-2. 
a

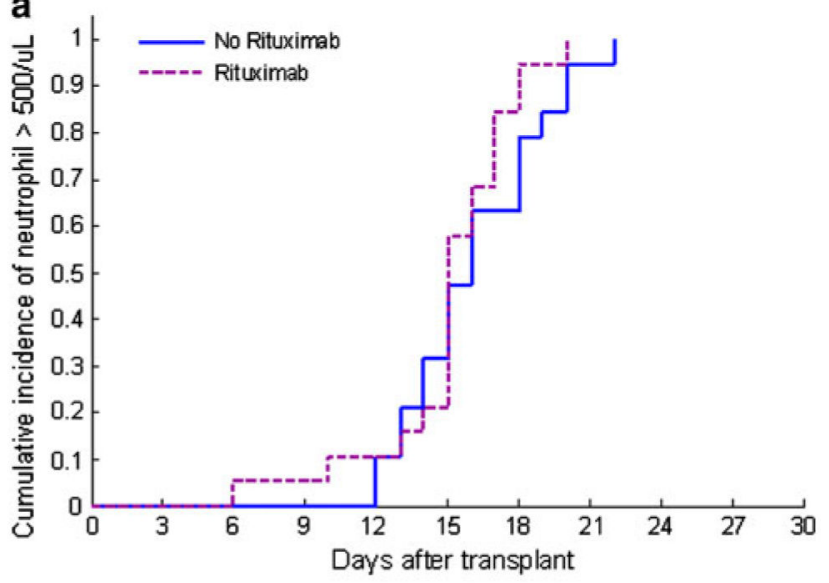

b

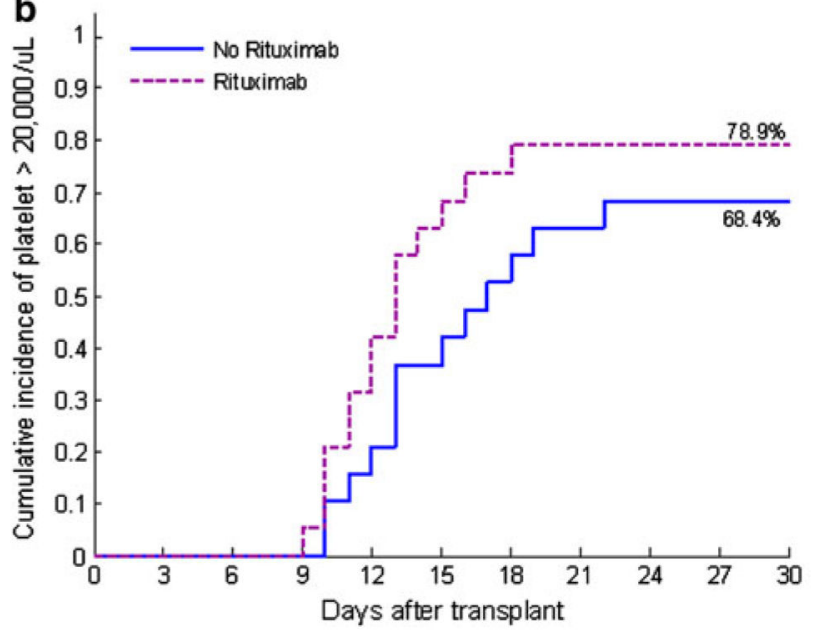

Fig. 1.

a Time to neutrophil engraftment, $p=0.31$ for comparison. b Time to platelet engraftment, $p$ $=0.2$ for comparison

Int J Hematol. Author manuscript; available in PMC 2017 December 06. 


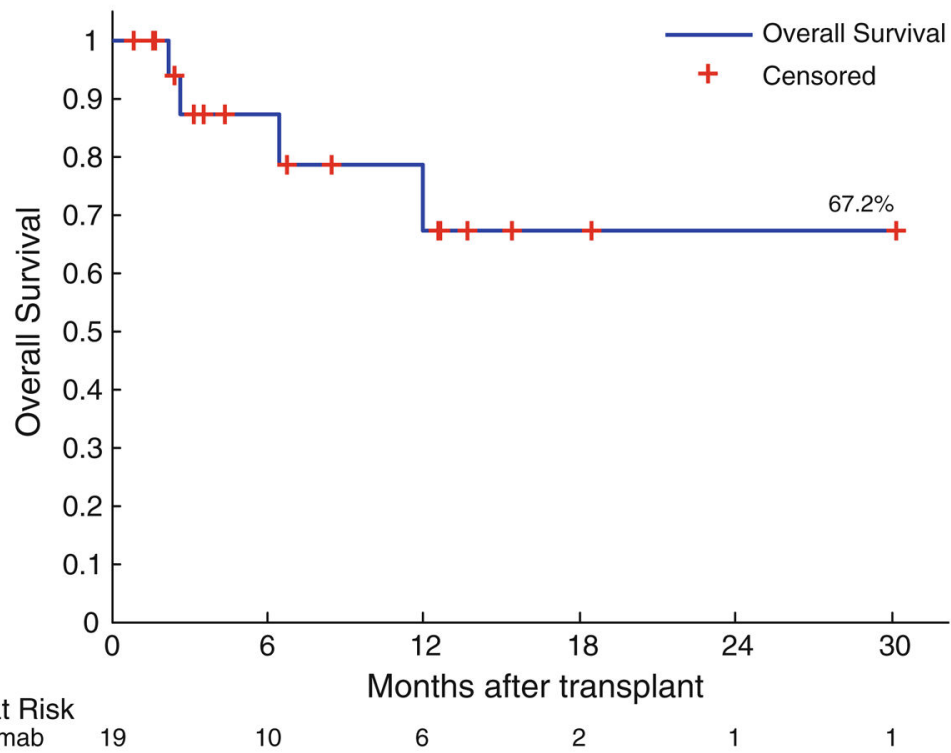

Fig. 2.

Kaplan-Meier plot of overall survival for t-IV Bu/flu + rituximab $(n=19)$ sample from date of AHCT (months) 


\section{Table 1}

Baseline characteristics

\begin{tabular}{|c|c|c|c|}
\hline & t-IV Bu/Flu + rituximab & t-IV Bu/Flu & $p$ value \\
\hline Age, median (range) & $56(35-68)$ & $47(27-64)$ & $p=0.03$ \\
\hline \multicolumn{4}{|l|}{ Gender } \\
\hline Male & 16 & 10 & $p=0.08$ \\
\hline Female & 3 & 9 & \\
\hline \multicolumn{4}{|l|}{ Diagnosis } \\
\hline Chronic lymphocytic leukemia & 8 & 5 & Diagnosis: $p=0.46$, remission status: $p=0.1$ \\
\hline CR1 & 0 & 1 & \\
\hline $\mathrm{CR} 2$ & 2 & 1 & \\
\hline PR2 & 4 & 1 & \\
\hline PR3 & 2 & 0 & \\
\hline REL1 & 0 & 1 & \\
\hline Refractory & 0 & 1 & \\
\hline Follicular lymphoma & 6 & 7 & \\
\hline $\mathrm{CR} 2$ & 0 & 3 & \\
\hline CR3 & 3 & 3 & \\
\hline PR1 & 1 & 0 & \\
\hline $\mathrm{PR} 2$ & 2 & 1 & \\
\hline Mantle cell lymphoma & 3 & 2 & \\
\hline $\mathrm{CR} 2$ & 0 & 1 & \\
\hline PR2 & 1 & 1 & \\
\hline PR3 & 1 & 0 & \\
\hline$>$ PR3 & 1 & 0 & \\
\hline Diffuse large cell lymphoma & 2 & 3 & \\
\hline PR2 & 0 & 2 & \\
\hline PR3 & 1 & 0 & \\
\hline$>$ PR3 & 1 & 0 & \\
\hline $\mathrm{CR} 2$ & 0 & 1 & \\
\hline Marginal zone lymphoma & 0 & 2 & \\
\hline PR2 & 0 & 2 & \\
\hline \multicolumn{4}{|l|}{ Donor relation } \\
\hline Matched related donor & 8 & 8 & $p=1.0$ \\
\hline Mismatched related donor & 1 & 0 & \\
\hline Matched unrelated donor & 7 & 7 & \\
\hline Mismatched unrelated donor & 3 & 4 & \\
\hline \multicolumn{4}{|l|}{ CMV serostatus (recipient/donor) } \\
\hline Negative/negative & 6 & 7 & $p=0.59$ \\
\hline Negative/positive & 4 & 1 & \\
\hline Positive/negative & 5 & 5 & \\
\hline Positive/positive & 4 & 6 & \\
\hline
\end{tabular}




\begin{tabular}{llll}
\hline & t-IV Bu/Flu + rituximab & t-IV Bu/Flu & $p$ value \\
\hline aGVHD prophylaxis regimen & & & \\
Tacrolimus/methotrexate & 11 & 14 & $p=0.59$ \\
Tacrolimus/mycophenolate mofetil & 6 & 4 & \\
Tacrolimus/sirolimus & 2 & 1 & \\
Conditioning regimen & & & \\
t-IV busulfan/fludarabine (t-IV Bu/Flu) & 15 & 15 & \\
t-IV busulfan/fludarabine (t-IV Bu/Flu) + ATG & 4 & 4 & \\
\hline
\end{tabular}




\section{Table 2}

Comparison of donor chimerism at days 30 and 90 post-transplant for t-IV Bu/Flu + rituximab versus t-IV $\mathrm{Bu} / \mathrm{Flu}$

\begin{tabular}{llll}
\hline & t-IV Bu/Flu + rituximab & t-IV Bu/Flu & $p$ value \\
\hline Day 30 (CD3) & $100 \%(10-100 \%)$ & $93 \%(75-100 \%)$ & 0.9 \\
Day 30 (CD33) & $100 \%(79-100 \%)$ & $100 \%(61-100 \%)$ & 0.86 \\
Day 30 (unsorted BM) & $98 \%(84-100 \%)$ & $98 \%(45-100 \%)$ & 0.54 \\
Day 90 (CD3) & $94 \%(73-100 \%)$ & $90 \%(62-100 \%)$ & 0.25 \\
Day 90 (CD33) & $100 \%$ & $100 \%(90-100 \%)$ & 0.35 \\
Day 90 (unsorted BM) & $95 \%(88-100 \%)$ & $98 \%(93-100 \%)$ & 0.25 \\
\hline
\end{tabular}

Int J Hematol. Author manuscript; available in PMC 2017 December 06. 
Table 3

Absolute lymphocyte count $(\mathrm{k} / \mu \mathrm{L})$ after HCT, presented as median (range) of values

\begin{tabular}{lccl}
\hline & t-IV Bu/Flu + rituximab & t-IV Bu/Flu & $\boldsymbol{p}$ value \\
\hline Day 0 & $0.05(0-62)$ & $0.1(0.01-2.3)$ & 0.5 \\
Day 30 & $0.79(0.06-3.26)$ & $0.85(0.09-2.2)$ & 1.0 \\
Day 90 & $0.375(0.13-0.97)$ & $0.65(0.21-3.5)$ & 0.03 \\
Day 180 & $0.56(0.03-1.17)$ & $0.59(0.08-1.9)$ & 0.6 \\
Day 360 & $0.84(0.12-3.01)$ & $1.06(0.34-2.26)$ & 0.7 \\
2 years & $1.425(1.2-1.98)$ & $1.4(0.97-8.13)$ & 0.84 \\
\hline
\end{tabular}


Table 4

Infectious complications after t-IV Bu/Flu + rituximab compared with t-IV Bu/Flu

\begin{tabular}{llll}
\hline & t-IV Bu/Flu + rituximab & t-IV Bu/Flu & $\boldsymbol{p}$ value \\
\hline Bacterial & $7 / 19$ & $10 / 19$ & 0.5 \\
Invasive fungal & $2 / 19$ & $5 / 19$ & 0.4 \\
Viral & $4 / 19$ & $4 / 19$ & 1 \\
\hline
\end{tabular}

